\title{
A Case of Urethral Reconstruction Using a Superficial Circumflex Iliac Artery
}

\author{
Kun-Woon Yoo, Hyun-Woo Shin, Hye Kyung Lee \\ Department of Plastic and Reconstructive Surgery, Myongji Hospital, Kwandong University College of Medicine, Goyang, Korea
}

\begin{abstract}
A radial forearm free flap has been conventionally used for urethral reconstruction. However, aesthetic and functional complications occur frequently at the donor site. The use of a superficial circumflex iliac artery perforator (SCIP) flap can resolve these disadvantages. Here, we report our case with a review of literature. A 69-year-old man visited our hospital with multiple contusions of the abdomen and genital amputation. After necrotic tissue debridement, the length of the residual corpus carvernosum was $1.5 \mathrm{~cm}$ and that of the corpus spongiosum and urethra was $1 \mathrm{~cm}$. For the reconstruction of the penis, a SCIP flap and anterolateral thigh free flap was performed. The primary closure was performed at the donor site. Three weeks postoperatively, the patient had a urethral foley catheter removed. The neourethra was functioning well without stricture. Four months postoperatively, the patient had no complications such as urethral stricture. A good recovery was also achieved with no aesthetic deficits at the donor site. SCIP flap is appropriate for urethral reconstruction. Because of its proximity to the recipient sites, it makes surgical preparation easier and the primary closure at the donor site available. It is also advantageous in that its location is almost unnoticeable.
\end{abstract}

Keywords Urethra / Skin transplantation / Free tissue flaps

\author{
Correspondence: Hyun-Woo Shin \\ Department of Plastic and \\ Reconstructive Surgery, Myongji \\ Hospital, Kwandong University \\ College of Medicine, 55 Hwasu-ro \\ 14beon-gil, Deokyang-gu, Goyang \\ 412-826, Korea \\ Tel: +82-31-810-6830 \\ Fax: +82-31-810-6837 \\ E-mail:drbrown0414@gmail.com
}

No potential conflict of interest relevant to this article was reported.

Received: 16 Jan $2012 \bullet$ Revised: 26 Mar 2012 • Accepted: 4 Apr 2012

pISSN: 2234-6163・ elSSN: 2234-6171 • http://dx.doi.org/10.5999/aps.2012.39.3.253・Arch Plast Surg 2012;39:253-256

\section{INTRODUCTION}

For urethral reconstruction in patients with genital amputation or congenital anomaly and those undergoing transgender surgery, a full-thickness skin graft or a radial forearm free flap have been frequently used. If a radial forearm free flap were to be used, this would make it possible to achieve urethral reconstruction more reliably than a skin graft while reducing the risk of infections or urethral stricture and bladder neck.

A radial forearm free flap is very useful in reconstructing the urethra, but it is disadvantageous in that aesthetic and functional complications occur frequently at the donor site. Therefore, ure- thral reconstruction using a superficial circumflex iliac perforator (SCIP) flap has the following additional advantages while retaining the excellent profile of using a flap.

A SCIP flap enables surgeons to achieve a one-stage reconstruction of a greater length of urethra. Besides, due to the proximity of the surgical sites, it makes the surgical preparation easier and the primary suture available at the donor site. It is therefore advantageous in minimizing the aesthetic and functional deficits of the donor sites [1].

Nevertheless, in very rare cases, urethral reconstruction has been performed using a SCIP flap. Here, we report such a case with a review of the literature. 


\section{CASE}

A 69-year-old man visited us with multiple contusions of the abdomen and genital amputation. For the management of the abdominal contusion and genital amputation, the patient underwent hematoma evacuation and primary repair in the Department of General Surgery and penis replantation in the De- partment of Urology. While monitoring the clinical course, the patient presented with a necrotic change in the penis (corpus carvenosum and urethra). The patient was therefore transferred to the Department of Plastic and Reconstructive Surgery. Following the transfer to us, the patient underwent a necrotic tissue debridement. In this patient, the length of the residual corpus carvernosum was $1.5 \mathrm{~cm}$ and that of the corpus spongiosum

\section{Fig. 1. Findings seen after the initial debridement}

(A) Lateral view after initial debridement. (B) Anterior view after the debridement.
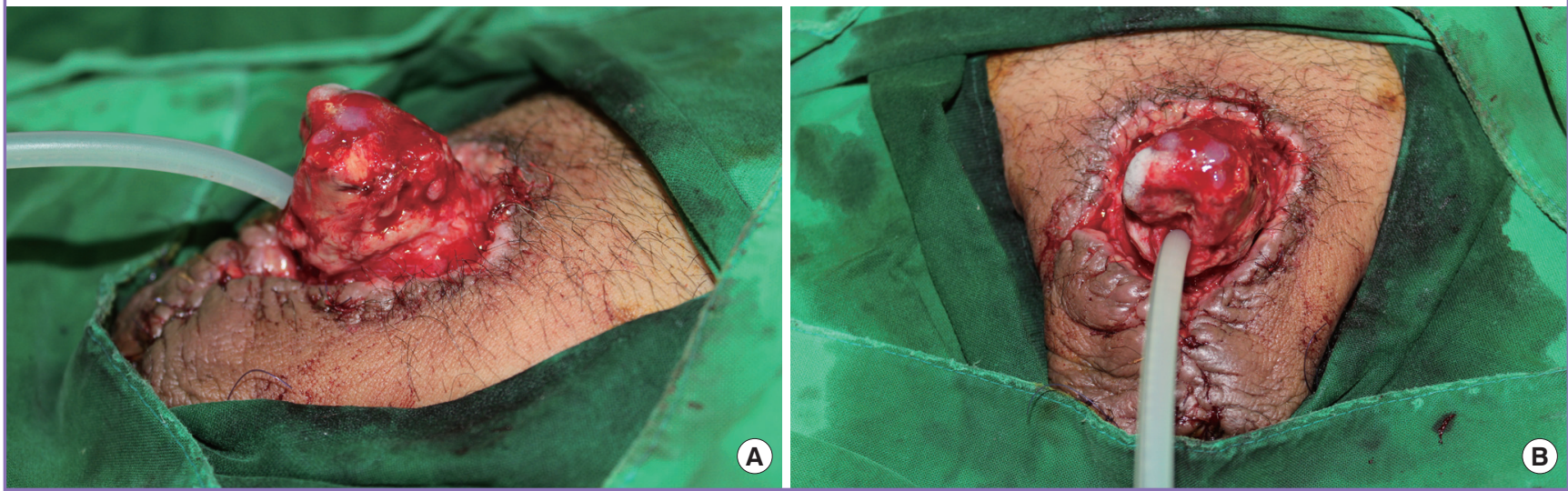

\section{Fig. 2. Intraoperative findings}

(A) Superficial circumflex iliac perforator (SCIP) flap design. (B) Urethral reconstruction with a SCIP flap. (C) Anterolateral thigh flap being inset. (D) The completion of the procedure.
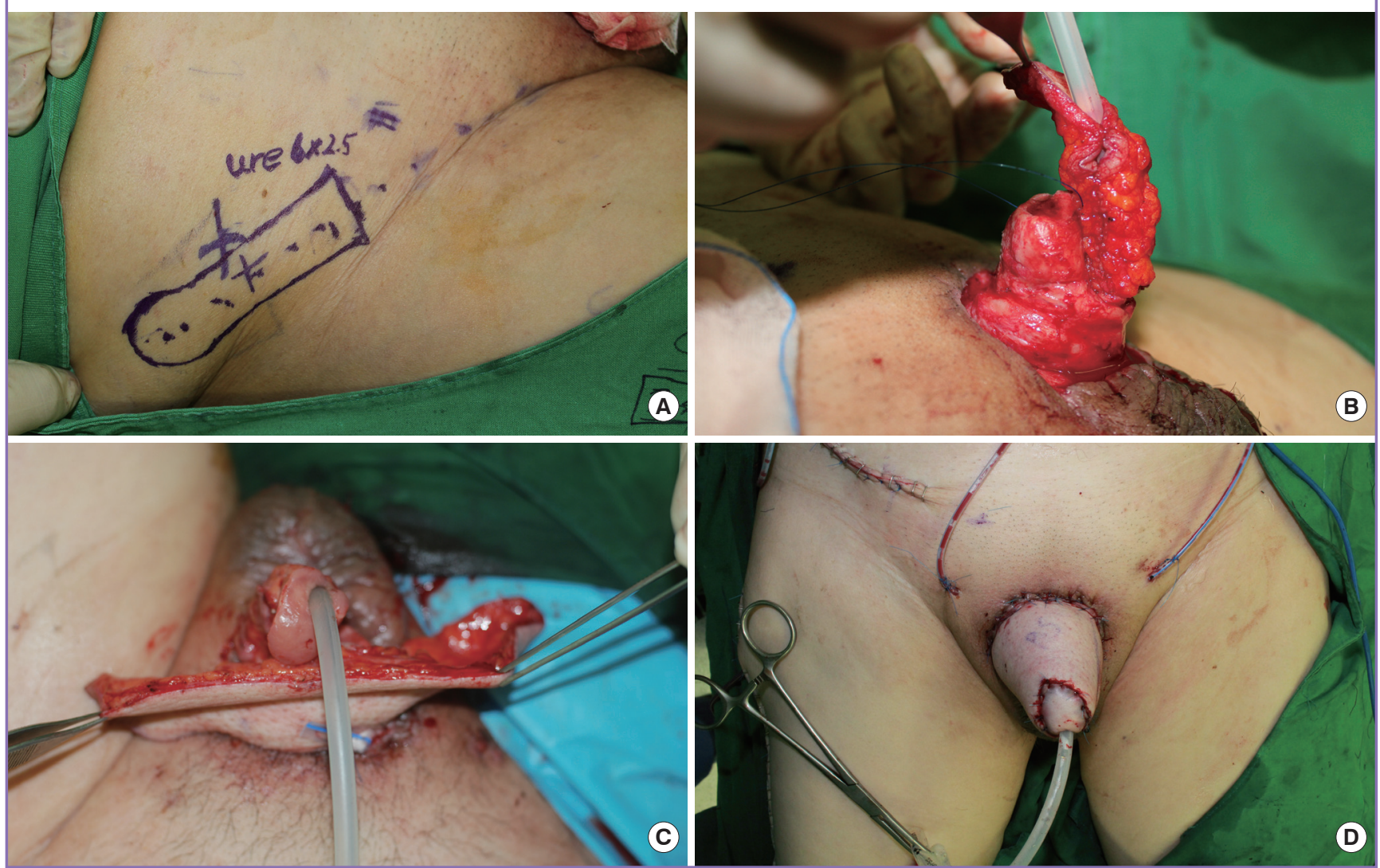
and urethra was $1 \mathrm{~cm}$. It was confirmed that the patient had no normal testicles and had urethral stenosis (Fig. 1).

The patient underwent dressings using betadine gauze every day for a week. This was followed by penile reconstruction under general anesthesia. Surgery was performed with the insertion of a foley catheter, where the granulation tissue and necrotized tissue were all removed. This was also accompanied by the dissection of the corpus spongiosum and urethra.

For the reconstruction of the urethra and glans, a SCIP flap of $6 \times 2.5 \mathrm{~cm}$ was elevated from the right inguinal area. For microsurgery, the superficial circumflex iliac artery perforator and one vena comitans were exposed as the donor vessels and the penile dorsal artery and one penile dorsal vein were exposed as the recipient vessels. This was followed by microanastomosis.

For reconstruction of the penile shaft, an anterolateral thigh (ALT) flap of $14 \times 6 \mathrm{~cm}$ was elevated. One descending branch of the left lateral circumflex femoral artery and two vena comitans served as the donor vessels. One deep circumflex iliac artery and two vena comitans served as the recipient vessels for the reconstruction (Fig. 2).

Both flaps had a warm ischemic time of 60 minutes. Primary closure was performed all of the donor sites.

Three weeks postoperatively, the patient had the urethral foley catheter removed. The neourethra was functioning well. Four months postoperatively, during monitoring of the clinical course, the patient had no complications such as urethral stricture. A good recovery was also achieved with no aesthetic deficits at the donor site (Fig. 3).

\section{DISCUSSION}

Penile reconstruction is difficult because of its characteristic shape. It is also difficult to achieve penile reconstruction to a satisfactory extent from an aesthetic perspective. This is a great challenge to plastic surgeons. With the use of a free flap based on the advancement of microsurgical techniques, great progress has been made.

In 1984, Chang and Hwang [2] successfully achieved a penile reconstruction using a radial forearm free flap. Since then, a radial forearm free flap has been used for the most ideal penile reconstruction outcomes. It has been reported, however, that a radial forearm free flap has such disadvantages as color mismatch, longterm atrophy, conspicuous donor site and potential morbidity of the radial artery sacrificed [3].

To overcome these disadvantages, various types of flaps have been considered $[4,5]$. An ALT flap was first designed by Song et al. [6] in 1984. Thereafter, it has been used to reconstruct various body organs.

In recent years, it has also been used for a penile reconstruction. If an ALT flap should solely be used, a persistent urethrocutaneous fistula or urethral stricture can arise in some cases [7]. In urethra reconstruction using a free flap, the thickness of the flap remains problematic. A radial forearm flap has therefore been reported to be an ideal flap. In the synchronous reconstruction of both the penis and urethra, a large donor scar has been reported to be problematic.

Harashina et al. [8] introduced the methods of urethra reconstruction using an ulnar forearm flap and penile reconstruction using a deltoid flap. This is advantageous in that noticeable donor scars can be minimized. But these methods also leave a scar on the arm. Therefore, further options for the reconstruction include using a SCIP flap and an ALT flap. Using a SCIP flap is useful for elevating a thinner flap. It is therefore appropriate for urethral reconstruction. In addition, due to the proximity of the surgical sites, it makes surgical preparation easier and the primary closure at the donor site can be easily achieved in most

Fig. 3. Postoperative findings

(A) Postoperative urination through the neourethra and neophallus. (B) Follow-up at postoperative 2 months.
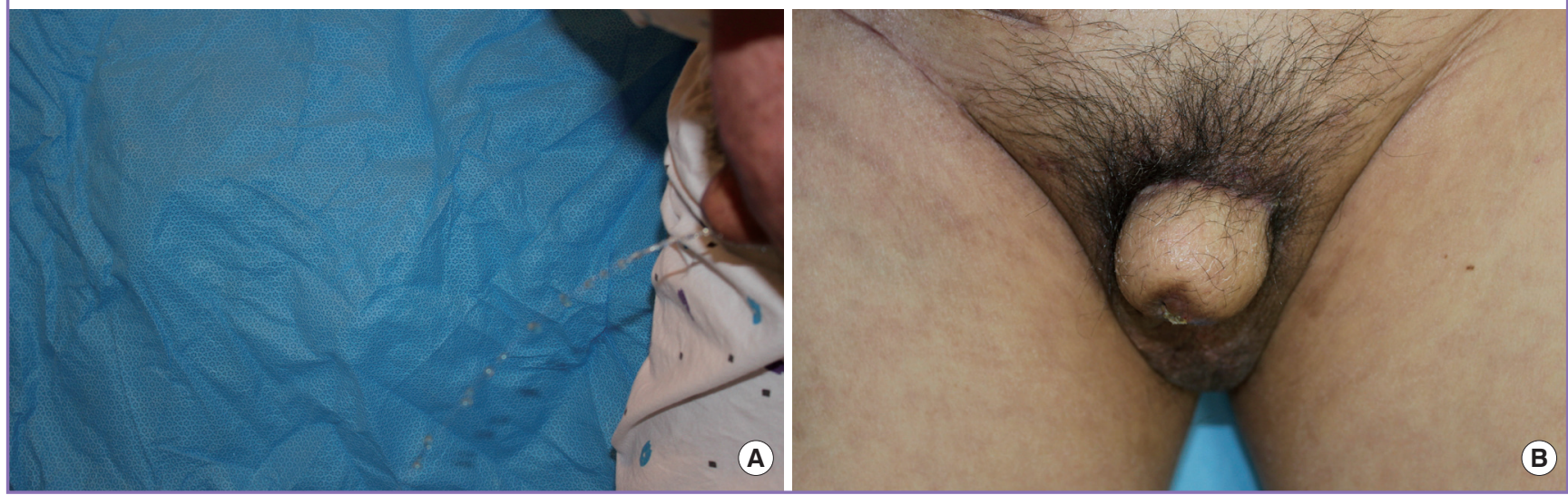
urethral reconstruction cases. It is also advantageous in that its location is almost unnoticeable.

Postoperatively, the patient had donor scars that were almost unnoticeable when wearing pants. Therefore, in contrast to a radial forearm flap the patient did not also have to concern himself with wrist scars when wearing short sleeved shirts.

In spite of these advantages, some limitations remain for penile and urethral reconstruction using a with dual free flap as in this case. The prolonged microvascular procedure time and difficulties in buried urethral flap monitoring are obvious limitations. In addition, the procedure is more influenced by the recipient vessel condition and any negative effects of the outer flap on the inner flap such as swelling and hematoma are disadvantages of this procedure.

Despite some limitations, our case indicates that penile reconstruction using a SCIP flap and an ALT flap had good functional and aesthetic outcomes, so we concluded that this procedure should not be taken as a gold standard for urethral and penile reconstructions but rather as a good alternative surgery technique.

\section{REFERENCES}

1. Koshima I, Nanba Y, Nagai A, et al. Penile reconstruction with bilateral superficial circumflex iliac artery perforator (SCIP) flaps. J Reconstr Microsurg 2006;22:137-42.

2. Chang TS, Hwang WY. Forearm flap in one-stage reconstruction of the penis. Plast Reconstr Surg 1984;74:251-8.

3. Lee GK, Lim AF, Bird ET. A novel single-flap technique for total penile reconstruction: the pedicled anterolateral thigh flap. Plast Reconstr Surg 2009;124:163-6.

4. Papadopulos NA, Schaff J, Biemer E. Usefulness of free sensate osteofasciocutaneous forearm and fibula flaps for neophallus construction. J Reconstr Microsurg 2001;17:407-12.

5. Hu ZQ Hyakusoku H, Gao JH, et al. Penis reconstruction using three different operative methods. Br J Plast Surg 2005; 58:487-92.

6. Song YG, Chen GZ, Song YL. The free thigh flap: a new free flap concept based on the septocutaneous artery. Br J Plast Surg 1984;37:149-59.

7. Lumen N, Monstrey S, Ceulemans P, et al. Reconstructive surgery for severe penile inadequacy: phalloplasty with a free radial forearm flap or a pedicled anterolateral thigh flap. Adv Urol 2008:704343.

8. Harashina T, Inoue Y, Takamatsu A, et al. Construction of penis with two free flaps. Ann Plast Surg 2002;49:302-6. 